\title{
LA INTERPRETACIÓN COMO TEMA DE FICCIÓN EN LOS RELATOS DE JULIO CORTÁZAR
}

\author{
Patricio GOYALDE PALACIOS
}

Centro Superior de Música del País Vasco - Musikene

La complejidad, diversidad y ambigüedad de los relatos de Julio Cortázar han suscitado numerosos problemas interpretativos entre quienes se han acercado a ellos con una intención crítica. La variedad de lecturas realizadas a propósito de su obra narrativa breve es realmente impresionante, hasta el punto de que dichos relatos constituyen un campo de prueba de los principios teóricos que sustentan las diferentes corrientes interpretativas ${ }^{1}$.

Ahora bien, a este interés de la crítica por la obra cortazariana se debe añadir el del propio autor por las cuestiones interpretativas; en efecto, Cortázar, quizá consciente de las dificultades de sus textos, discute con diversos intérpretes algunas de sus lecturas — véanse, por ejemplo, su respuesta a Julio Cortázar o la crítica de la razón pragmática de Juan Carlos Curuchet, en Cortázar (1973), o «Noticias de los Funes», en Último

\footnotetext{
${ }^{1}$ Para una visión global de esta cuestión puede consultarse Goyalde (2001).
} 
Round - o ironiza sobre lo absurdo de algunas polémicas interpretativas, la pedantería del lenguaje académico y la necesidad de las explicaciones -así en «Texturologías» y «Destino de las explicaciones» de Un tal Lucas - y, desde el punto de vista de la creación literaria, introduce el tema en su propia ficción narrativa.

En este trabajo me propongo tratar el último aspecto señalado: la presencia de los problemas interpretativos y de las propuestas críticas como clave de lectura de algunos de sus relatos. Para ello, me centraré en dos textos que, a mi entender, son los más significativos en relación con esta temática: «El perseguidor», un cuento que leeré como una reflexión sobre la posibilidad del discurso crítico sobre la música, que podría ser considerada, en este caso, como paradigma del arte, y «Los pasos en las huellas», relato centrado en la interpretación literaria, que estudiaré como una crítica a la hermenéutica de autor y al intencionalismo, y como un ejemplo del efecto de la interpretación sobre el propio intérprete.

\section{1. «EL PERSEGUIDOR»: LA POSIBILIDAD DEL DISCURSO CRÍTICO SOBRE EL ARTE}

«El perseguidor» (Las armas secretas, 1959) es un relato protagonizado por un músico de jazz, Johnny Carter - personaje de ficción basado en la figura del saxofonista Charlie Parker, máximo exponente del estilo bebop que revolucionó la música de jazz de los años 40 - y por un crítico, Bruno, autor de un estudio biográfico y musical sobre Carter, que trata por momentos de entender su problemática figura y que ejerce el papel de contrapunto a los excesos y a la genialidad del artista $^{2}$.

\footnotetext{
2 Según sus declaraciones, Cortázar había escuchado discos de Parker en Argentina, pero el punto de arranque de su relato fue la lectura de un artículo de la revista Jazz Hot, en el que se hacía una semblanza biográfica de la figura de Charlie Parker tras su muerte. Cortázar alteró el nombre, si bien dedicó el relato a la memoria del saxofonista, y tomó del artículo algunas de las anécdotas reales, ubicándolas en París en lugar de Nueva York (González Bermejo, 1978: 107). Además en el texto se citan a varios críticos y productores, que son figuras reales y no de ficción como Bruno: Charles Delaunay, Hugues Panassié, André Hodeir, Leonard Feather y Boris Vian. Cortázar establece un nexo con la realidad jazzística del momento de escritura del relato, al mencionar la polémica que rodeó a la música de Parker, especialmente en Francia donde suscitó enconados debates.
} 
Como casi todos los textos de Cortázar, este cuento admite múltiples lecturas: nos podríamos centrar en la problemática metafísica del tiempo y del espacio que constantemente aparece en las oscuras reflexiones de Johnny Carter; sería posible leer el relato en clave sociológica, analizando la incomprensión de la sociedad ante el artista genial; en clave religiosa, descifrando las referencias bíblicas que se repiten en el texto, etc.

En el análisis que sigue, opto por restringir mi lectura a las relaciones entre el músico y el crítico, porque creo que de ella puede extraerse, además de una reflexión sobre el papel de la crítica, una interrogación sobre la posibilidad del discurso crítico sobre la música.

\subsection{Representación y parodia de la crítica}

El relato se articula como una constante oposición entre la figura del músico creador y la del crítico, entre la genialidad del saxofonista y el pragmatismo del comentarista y biógrafo.

En efecto, Johnny Carter responde a la figura del músico genial que, sin embargo, vive en una situación miserable, en una lúgubre habitación de hotel, enfermo y sin medios económicos y, por si esto fuera poco, enganchado al mundo de la droga. Su comportamiento excéntrico con los músicos que lo acompañan, en el estudio de grabación, y su relación destructiva con los objetos — prende fuego a la habitación de su hotel; pierde o rompe su saxofón en numerosas ocasiones - lo convierten en un personaje límite, que hace estallar todas las situaciones a las que su profesión y vida personal lo enfrentan.

El crítico es, por contraposición, un puritano que muestra su horror ante el desorden moral y material en el que vive inmerso Johnny, que se lamenta de la buena música y de las posibles grabaciones que se están perdiendo a causa de esa vida marcada por la miseria y las drogas. Bruno se sitúa en un mundo ajeno al de la fatalidad y el desconcierto de los músicos del relato, a los que define como «ángeles enfermos» (Cortázar, 1994: 238).

Podríamos decir, en términos cortazarianos, que Johnny Carter es el cronopio que busca trascender lo aceptado como real - aun a riesgo de perderlo todo, incluida su propia cordura-, mientras que el crítico Bruno es el fama que se agarra a la realidad inmediata, a su cómoda vida de 
seguridad y prestigio 3 ; el primero es el ser intuitivo, que no logra comprender lo que los demás consideran real, mientras el segundo es el ser racional que se aferra al orden establecido.

Además de esta oposición general, Cortázar traza un despiadado retrato del crítico, que roza la caricatura, sobre todo en su papel de buen samaritano que se preocupa por el artista perdido en sus desvaríos, que le entrega dinero, a través de su compañera, en los momentos más difíciles. Sus cuidados hacia el músico esconden una buena carga de egoísmo, de intentar salvar la idea que él se ha construido del músico, de temor ante alguna declaración imprudente de Johnny que acabe desmontando todo su sistema teórico sobre el jazz moderno, tan elogiado y plasmado en su exitosa biografía. El colmo de esta actitud interesada se constata al final del cuento, cuando, tras la muerte de Johnny, Bruno se congratula de haber tenido tiempo de incluir una nota necrológica en la última edición de su libro.

El relato constituye así un duro alegato contra el papel de la crítica y, de forma más precisa, contra aquellos críticos que, incapaces de comprender el mundo del músico genial, se compadecen caritativamente de él, escondiendo tras la apariencia filantrópica, su propio interés, egoísmo y una buena dosis de cobardía 4 .

Sin embargo, y como ocurre en la mayor parte de los textos cortazarianos, los protagonistas no son personajes monolíticos, de una pieza, sino que acumulan contradicciones y, por momentos, el lector se sorprende de las múltiples caras que pueden presentar. En este relato, la figura de

3 La oposición entre los dos términos, que dará título a su conocido libro Historias de cronopios y de famas (1962), tiene su origen precisamente en un artículo de Cortázar dedicado a Louis Armstrong, titulado «Louis, enormísimo cronopio». Esta crónica de un concierto parisino del famoso músico de jazz, publicada en 1952 en la revista Buenos Aires Literaria, y recogida luego en La vuelta al día en ochenta mundos, es el primer texto escrito en el que Cortázar utiliza el término cronopio. No deja de ser significativo que esta denominación, connotada siempre positivamente por el autor, aparezca en su obra, por vez primera, relacionada con un músico de jazz.

${ }^{4}$ El propio crítico se muestra implacable: «En el fondo somos una banda de egoístas, so pretexto de cuidar a Johnny lo que hacemos es salvar nuestra idea de él, prepararnos a los nuevos placeres que va a darnos Johnny, sacarle brillo a la estatua que hemos erigido entre todos y defenderla cueste lo que cueste. El fracaso de Johnny sería malo para mi libro (de un momento a otro saldrá la traducción al inglés y al italiano), y probablemente de cosas así está hecha una parte de mi cuidado por Johnny» (Cortázar, 1994: 240). 
Bruno, caricaturizada, como hemos señalado anteriormente, como un falso samaritano, presenta una vertiente lúcida que añade al relato una dimensión que va más allá de la parodia y de la ridiculización del papel de la crítica.

\subsection{La función crítica como un satélite de la creación}

Bruno defiende, en un intento de argumentar teóricamente su propio rol vital, la necesidad del discurso crítico, pues los creadores, y entre ellos los músicos, «son incapaces de extraer las consecuencias dialécticas de su obra, postular los fundamentos y la trascendencia de lo que están escribiendo o improvisando» (Cortázar, 1994: 258).

Johnny es, según la visión de Bruno, un pobre diablo dotado del don de la creación, sin la menor conciencia de las dimensiones de su obra. Por ello, la función del discurso crítico será explicitar, por medio de la palabra, tanto los principios en los que se fundamenta la genialidad del artista como el valor de su obra, aspectos que, según el crítico cortazariano, aquél desconocería.

A pesar de esta aparente seguridad, Bruno duda continuamente de su propio papel y comprende sus limitaciones ante la personalidad del artista 5 ; con una lucidez que no lo abandona casi nunca, piensa melancólicamente que el crítico no es sino «el triste final de algo que empezó como sabor, como delicia de morder y mascar» (Cortázar, 1994: 229) y que, en definitiva, no es más que «ese hombre que sólo puede vivir de prestado, de las novedades y las decisiones ajenas» (1994: 253).

La función crítica aparece así como un apéndice de la creadora, como una tarea derivada que en ningún caso puede alcanzar una autonomía y que parece abocada a la categoría de resto amargo tras la degustación del arte musical. El crítico no sería sino un parásito, un satélite, empleando la

5 «Soy un crítico de jazz lo bastante sensible como para comprender mis limitaciones, y me doy cuenta de que lo que estoy pensando está por debajo del plano donde el pobre Johnny trata de avanzar con sus frases truncadas, sus suspiros, sus súbitas rabias y sus llantos» (Cortázar, 1994: 229). Esto no es obstáculo para que manifieste su envidia por el saxofonista y por su capacidad de trascender la realidad: «[...] envidio a Johnny, a ese Johnny del otro lado, sin que nadie sepa qué es exactamente ese otro lado» (Cortázar, 1994: 238). 
terminología del relato, cuya existencia es dependiente y subordinada en relación con la creación.

\subsection{La posibilidad del discurso crítico}

La lectura que realizamos de este relato nos conduce a una cuestión central: ¿es posible el discurso crítico sobre el arte?; y si la respuesta fuera afirmativa, ¿qué discurso?

La respuesta del texto, representada por las reflexiones de Bruno, es ambivalente. Por un lado, como ya se ha señalado, considera necesaria la tarea crítica como una forma de racionalizar lo que no es sino intuición en el mundo artístico. Sin embargo, por otra, el músico es un misterio indescifrable que no tiene explicación y que cuestiona las certezas del crítico.

La creación artística de Johnny Carter es inseparable de su búsqueda, de sus incomprensibles reflexiones sobre el tiempo, de sus delirios visionarios, que constituyen una vertiente metafísica que escapa al control del crítico. Incluso en un momento del cuento, Bruno reflexiona sobre la importancia de conseguir que Johnny retorne a la realidad, pero entonces todas sus categorías entran en crisis para concluir que no es posible que la realidad consista en ser crítico de jazz (Cortázar, 1994: 248).

De acuerdo con mi lectura, el relato propone que la crítica se reduzca a un discurso descriptivo, que se limite a «sancionar comparativamente» (Cortázar, 1994: 241), pero niega la posibilidad del discurso interpretativo. Por eso, el libro de Bruno solamente teoriza sobre la música y el papel renovador del saxofonista en el panorama jazzístico; sobre sus aportaciones al lenguaje musical predominante hasta entonces y el lugar que, en consecuencia, ocupa en la historia del jazz. No consigue ir más allá, por lo que renuncia a una posibilidad interpretativa latente en las actitudes y ocurrencias del músico: la reflexión sobre el sentido de la música de jazz como una búsqueda trascendente. El crítico intuye continuamente que la figura de Johnny «encubre otra cosa» (Cortázar, 1994: 241), que es realmente lo único importante, pero manifiesta su incapacidad para acceder a ello y trasmitirlo por medio de la palabra.

Bruno cree que la genialidad es única $y$, en consecuencia, piensa que Johnny es un genio singular en relación con otros como Picasso, Einstein o Stravinsky; pero a la vez siente que su diferencia «es secreta, irritante por lo misteriosa, porque no tiene ninguna explicación» (Cortázar, 1994: 
248). La derrota del crítico ante el enigma de la figura del músico se convierte, a efectos del discurso crítico, en un pragmatismo descriptivo que desconcierta al propio Johnny que no se reconoce en el libro: «Al principio me pareció que hablabas de algún otro [...] y yo me preguntaba ¿pero éste soy yo?» (Cortázar, 1994: 262).

Como el propio crítico señala, el título del relato adquiere así un nuevo sentido: el perseguidor no es tanto Bruno, que va tras las huellas del genio intentando comprender su arte y sus comportamientos, sino el propio Johnny, quien a través de la música parece querer llegar a un lugar más allá, desconocido incluso para sí mismo. «Nadie puede saber qué es lo que persigue Johnny, pero es así, está ahí, en Amorous [una de sus grabaciones], en la marihuana, en sus absurdos discursos sobre tanta cosa [...]» (Cortázar, 1994: 250).

La visión que da el relato sobre la función crítica es ciertamente escéptica: los continuos desencuentros entre las figuras del crítico y del músico constituyen un reflejo de la dificultad de articular un discurso que, más allá de lo descriptivo, corra el riesgo de interpretar y comprender la actividad artística y musical como un hecho que trasciende a la obra creada.

\section{2. «LOS PASOS EN LAS HUELLAS»: LA CRÍTICA A UNA HERMENÉUTICA DE AUTOR}

«Los pasos en las huellas» (Octaedro, 1974) es un relato de carácter metaliterario, pues habla de la literatura, y, precisando un poco más, diría que metacrítico, ya que su protagonista propone un modelo interpretativo de búsqueda del sentido que nos remite a una hermenéutica de autor. Así pues, el hilo conductor que va a guiar mi exposición es la propuesta interpretativa que deduzco de la lectura del texto, y que puede ser sintetizada en pocas palabras: el crítico de Cortázar propone un modelo hermenéutico que, en la dialéctica autor-obra-lector, se decanta hacia el primero de los términos, por lo que la interrogación sobre el sentido de la obra se convierte en una indagación sobre la figura del autor, su intención oculta y sus vicisitudes biográficas.

La interpretación así concebida presupone que la función del intérprete es reconstruir el mundo original de la obra, equiparándose con el autor, 
con el fin de poner de manifiesto sus intenciones, no sólo las conscientes, sino también las supuestamente inconscientes; es célebre la fórmula repetida por autores de la hermenéutica romántica: se trata de «comprender a un autor mejor de lo que él mismo se habría comprendido» (Gadamer, 1996: 246).

Por lo tanto, el recorrido que propongo trata de averiguar de qué manera se inscribe el relato en la opción interpretativa que acabo de mencionar; de qué forma entiende el intérprete que debe realizarse la búsqueda del sentido de la obra; cómo se relaciona con el autor, con su misterio y su biografía, y, en fin, cuál es la propuesta de definición del sentido de la obra literaria que, de forma más o menos explícita, defiende el personaje de este cuento.

\subsection{La crítica como tema literario}

«Los pasos en las huellas» es un relato cuya ficción se centra en el tema de la interpretación literaria, por medio de un protagonista que convierte la búsqueda del sentido de una obra en obsesión vital, lo que pone al descubierto los presupuestos teóricos en los que fundamenta su tarea interpretativa.

El crítico Jorge Fraga decide estudiar la vida y la obra del poeta Claudio Romero, con el fin de encontrar un «sentido más personal y quizá más profundo» (Cortázar, 1974: 26) que el que habían difundido hasta entonces artículos laudatorios y estudios entusiastas en los que «el lirismo suplía las ideas» (Cortázar, 1974: 25). Por consiguiente, el punto de partida es el de un crítico literario que se enfrenta a una obra con el fin de interpretarla de una manera novedosa, más rigurosa y sistemática, de tal forma que salga a la luz el verdadero sentido de los textos analizados.

El camino para lograr tal objetivo aparece plagado de dificultades y obstáculos que convierten el recorrido crítico en un vaivén zigzagueante, en el que el protagonista ha de confrontar sus posiciones con nuevos e inesperados descubrimientos que trastocan sus hipótesis de trabajo y se convierten en móvil de la acción narrativa. El personaje de Cortázar acosa a la hija de Susana Márquez, con el fin de que le proporcione nuevos datos sobre la relación de su madre con el poeta estudiado, y se ve obligado a revisar sus posiciones ante el descubrimiento de unas cartas ocultas que, de acuerdo con su punto de vista, revelan la verdad sobre la figura y la 
obra del poeta Claudio Romero 6 . Por ello, la conversión del problema interpretativo en tema literario no está exenta de un cierto escepticismo en relación con el objetivo que se propone el personaje, ya que resulta manifiesta la dificultad de la aprehensión de un significado que se pretende plasmar de forma discursiva en un nuevo texto. Así, el lector observa los movimientos del personaje principal y la inevitable provisionalidad de toda conclusión interpretativa basada en la investigación biográfica.

\subsection{Una hermenéutica de autor}

La propuesta interpretativa que realiza el protagonista centra su búsqueda del significado en el autor de la obra analizada; de esta forma la tarea crítica se convierte en una indagación sobre las intenciones ocultas del autor o sobre determinados hechos biográficos que, supuestamente, aclararían las dificultades que surgen en la comprensión de la obra.

El personaje de Cortázar trata de desentrañar el sentido de la obra de un autor ya muerto, por lo que su presupuesto inicial es indagar en su biografía, para poner de manifiesto la indisoluble unidad entre vida y obra, y la necesidad de recurrir a la primera con el fin de comprender la segunda. En efecto, la primera frase del relato nos informa de que Jorge Fraga, al cumplir los cuarenta años, «decidió estudiar la vida y la obra del poeta Claudio Romero» (la cursuva es mía); su propósito, continúa el narrador, va más allá de lo filológico y lo estilístico y pretende llegar a una noción más profunda de la biografía, en la que «el hombre, la tierra y la obra debían surgir de una sola vivencia» (Cortázar, 1974: 26).

A partir de este principio básico, el crítico comienza su indagación en la vida del poeta, descubriendo sus cartas a Susana Márquez, las cuales alumbran una concepción del amor y del mundo que se refleja de forma

\footnotetext{
6 El relato de Cortázar va encabezado por el siguiente epígrafe: «Crónica algo tediosa, estilo de ejercicio más que ejercicio de estilo de un, digamos, Henry James que hubiera tomado mate en cualquier patio porteño o platense de los años veinte» (1974: 25). Lógicamente, a partir de aquí, son posibles múltiples lecturas intertextuales relacionando nuestro cuento con relatos de James; por ejemplo, en «Sir Dominick Ferrand» aparece el motivo de las cartas desconocidas, que sacan a la luz aspectos ocultos de la personalidad de un prohombre de la patria, que, de esta manera, queda desmitificado, algo similar a lo que ocurre con el poeta de «Los pasos en las huellas».
} 
notoria en su poesía, convirtiéndose aquéllas en la clave interpretativa de la nueva visión que Fraga pretende mostrar.

El crítico de Cortázar quiere comprender la obra desde la vida y, precisando un poco más, desde la vivencia, es decir, desde aquellos hechos vividos que, por su efecto, adquieren un significado duradero (Gadamer, 1996: 97); de esta forma, se sitúa en la órbita interpretativa del siglo XIX, época en la que la vivencia se convierte en el «concepto valorativo dominante» (Gadamer 1996: 118).

La propuesta interpretativa que realiza el protagonista conforma una auténtica declaración programática de los principios que guían a aquellos críticos cuyo centro de interés, más allá de la obra misma o de su incidencia en el lector, reside únicamente en el autor. En el relato de Cortázar, la crítica se convierte en una persecución, puesto que Jorge Fraga asedia a la hija de Susana Márquez, con el fin de ir obteniendo, en diversas entregas, las cartas que el poeta envió a ésta última, documentos que sacan a la luz nuevos detalles vivenciales del poeta, que se suponen claves ocultas del verdadero significado de su poesía.

Como veremos a continuación, esta concepción del crítico como un perseguidor, como un bucanero que saquea las fuentes de información en busca del tesoro escondido, implica una concepción del significado que se encuentra en la base de las categorías hermenéuticas de nuestro protagonista.

\subsection{El significado: ese misterio oculto}

Esta opción hermenéutica, que acabamos de explicar, supone una concepción del significado de la obra literaria como un misterio oculto que debe ser desvelado; de este modo, la labor del crítico se reduce a un descubrimiento, a la resolución de un acertijo, con el que pretende en definitiva, como señala Iser, reclamar la atención del público por su habilidad para sustraer del texto algo que se encontraba encubierto (Iser, 1987: 20-21).

El crítico de este relato cree que la poesía de Claudio Romero contiene unas claves ocultas, un misterio, que su investigación pretende desvelar: «Cuando hablaba de sus poemas con alumnos o amigos, Fraga llegaba a preguntarse si el misterio no sería en el fondo lo que prestigiaba esa poesía de claves oscuras, de intenciones evasivas» (Cortázar, 1974: 26). La aparición de unas cartas desconocidas, de una documentación inédita, se convierte en el núcleo central de la información, a partir de la cual 
nuestro crítico pretende revelar una nueva interpretación para una poesía de la que «casi nada se sabía de su sentido más personal y quizá más profundo» (Cortázar, 1974: 26).

Sin embargo, el relato, también en contra de la perspectiva del personaje, pone de manifiesto la dificultad de llegar a ese sentido oculto y la facilidad con la que el discurso crítico suele caer en las trampas que su propio método —o, en ocasiones, el azar — le puede tender: Fraga lee las primeras cartas con la convicción de haber descubierto una nueva cosmovisión del poeta Romero, que transformará la interpretación crítica de sus textos: «[...] todo coincide, todo se ajusta: ahora no hay más que escribirlo» (Cortázar, 1974: 34).

Su primer ensayo, Vida de un poeta argentino, constituye un sorprendente éxito editorial, la concesión del Premio Nacional y la posibilidad de obtener un cargo de agregado cultural en una embajada europea, antigua aspiración del personaje; ahora bien, su propia metodología pone de relieve sus carencias y dificultades: tras la escritura de su exitoso ensayo interpretativo, aparece una nueva carta inédita que echa por tierra todas sus hipótesis y hace recapacitar al crítico, hasta el punto de que en el discurso de recepción del Premio Nacional ofrece una visión absolutamente opuesta a la de su primer escrito, provocando el consiguiente escándalo entre el público que esperaba un discurso mitificador del poeta, en línea con la publicación primera del crítico.

No me interesan aquí las connotaciones sociológicas del relato, ni la crítica de la autocomplacencia del público, ni de la capacidad de mitificación y desmitificación que puede tener el quehacer interpretativo, aspectos todos ellos presentes en el texto de Cortázar. A mi entender, el relato tiene también interés en cuanto que cuestiona, quizá sin pretenderlo, la idea de un significado oculto que puede ser desvelado a través de la investigación de las fuentes: la aparición de una carta inédita desbarata todas la hipótesis críticas, basadas, a su vez, en la correspondencia inédita descubierta en primer lugar; pero, ¿quién asegura al intérprete que no pueda aparecer una nueva documentación desconocida, que vuelva del revés todas sus seguridades, en un proceso que, al menos como hipótesis, hay que suponer infinito?

Este texto cuestiona esa visión del significado de la obra literaria como un misterio oculto que debe ser descubierto: el fracaso de su personaje así lo atestigua. 


\subsection{Interpretar y aplicar: el efecto sobre el intérprete}

Gadamer, al analizar el problema hermenéutico, señala la importancia de la aplicación, es decir, el efecto que produce la interpretación sobre el intérprete: «[...] en la comprensión siempre tiene lugar algo así como una aplicación del texto que se quiere comprender a la situación actual del intérprete» (Gadamer, 1996: 379). La aplicación, continúa, no es una parte eventual del fenómeno de la comprensión, sino que la determina de forma global, ya que el intérprete no puede «ignorarse a sí mismo» ni olvidar «la situación hermenéutica concreta en la que se encuentra»: «[...] en último extremo toda comprensión es un comprenderse (Gadamer, 1996: 326 y 396).

El relato que aquí nos ocupa constituye un buen ejemplo de lo que acabamos de mencionar: su personaje principal experimenta el efecto de la interpretación y se produce una aplicación de la comprensión del texto a la situación del crítico, puesto que Jorge Fraga, a partir de la interpretación de la poesía de Romero, es consciente de los numerosos paralelismos entre su posición y la del autor analizado: ambos han sido «hermanos en la farsa, en la mentira esperanzada de una ascensión fulgurante, hermanos en la brutal caída que los fulminaba y destruía» (Cortázar, 1974: 45-46).

El personaje de «Los pasos en las huellas» llega a la conclusión de que su crítica biográfica no es sino una autobiografía disimulada, en la que resulta especialmente difícil que su sombra no pise los pies del poeta interpretado (Cortázar, 1974: 28). De esta manera, el relato señala otro de los elementos de la hermenéutica de autor: la búsqueda en los aspectos recónditos e inéditos de una biografía, con el fin de comprender la obra, implica inevitablemente una proyección del intérprete, que determina la elección de los detalles vitales del creador, que, en principio, podrían ser inagotables.

El relato de Cortázar es un magnífico ejemplo de esta práctica llevada a su extremo: difícilmente se puede encontrar una manera mejor de equipararse con el autor que acabar comprendiendo que el intento de crítica biográfica no es más que una autobiografía disimulada. El lector, al terminar el relato, está legitimado para preguntarse si ciertamente la ascensión y caída del poeta Romero en la más absoluta de las miserias morales - clave interpretativa que propone Fraga - no es sino una invención del crítico que proyecta su situación vital en la del poeta analizado; al menos, 
el efecto aplicativo de la interpretación sirve al crítico para cuestionar, no sólo su teoría, sino también su posición moral, sus anhelos por promocionarse y conseguir los honores, su fulgurante ascenso y caída en el abismo (Cortázar, 1974: 45-46).

\section{CONCLUSIÓN}

El fracaso de los protagonistas de ambos relatos pone de manifiesto una visión escéptica del autor sobre la posibilidad de la interpretación y del discurso crítico. En el primer caso, Bruno es incapaz de comprender el fenómeno artístico y se resigna a una crítica de carácter descriptivo, cuyo notable éxito de público no esconde su frustración ante la impenetrabilidad de la música de un genio que revolucionó las categorías establecidas del jazz de su época. En el segundo relato, Fraga experimenta una hermenéutica de autor que fracasa por la trampas del propio método; sin embargo, el crítico, en cierta medida, se redime por el efecto causado sobre su persona por la tarea interpretativa.

En los dos relatos analizados, los críticos son presentados como perseguidores de los creadores; en un caso, por su relación en vida; en el otro, por la inmersión que realiza el intérprete en la biografía del poeta. El fracaso antes mencionado quizá refleje las dudas de Cortázar hacia la interpretación concebida como una simbiosis con el productor de la obra artística, en este caso con el músico y el escritor, una situación causada por la dificultad de estos críticos para experimentar la distancia y elaborar un discurso autónomo con respecto al autor. 


\section{REFERENCIAS BIBLIOGRÁFICAS}

CORTÁZAR J. (1973). «La agarrada a patadas o el despertar de los monstruos o más sobre dados y ratitas o la respuesta del involuntario pero vehemente responsable». Cuadernos Hispanoamericanos 275, 223-229.

- (1974). Octaedro. Madrid: Alianza.

- (1994). Cuentos completos/1. Madrid: Alfaguara.

GADAMER, H. G. (1996). Verdad y método I. Fundamentos de una hermenéutica filosófica. Salamanca: Sígueme, $6^{\mathrm{a}}$ ed.

GonZÁlez Bermejo, E. (1978). Conversaciones con Cortázar. Barcelona: Edhasa.

GOYALDE, P. (2001). La interpretación, el texto y sus fronteras. Estudio de las interpretaciones críticas de los cuentos de Julio Cortázar. Madrid: UNED.

ISER, W. (1987). El acto de leer. Madrid: Taurus. 